Business Cases 
Andreas Taschner

\section{Business Cases}

Ein anwendungsorientierter Leitfaden

2., überarbeitete Auflage

算 Springer Gabler 
Andreas Taschner

ESB Business School Reutlingen

Reutlingen, Deutschland

Die Deutsche Nationalbibliothek verzeichnet diese Publikation in der Deutschen Nationalbibliografie; detaillierte bibliografische Daten sind im Internet über http://dnb.d-nb.de abrufbar.

\section{Springer Gabler}

(C) Springer Fachmedien Wiesbaden 2008, 2013

Dieses Werk einschließlich aller seiner Teile ist urheberrechtlich geschützt. Jede Verwertung, die nicht ausdrücklich vom Urheberrechtsgesetz zugelassen ist, bedarf der vorherigen Zustimmung des Verlags. Das gilt insbesondere für Vervielfältigungen, Bearbeitungen, Übersetzungen, Mikroverfilmungen und die Einspeicherung und Verarbeitung in elektronischen Systemen.

Die Wiedergabe von Gebrauchsnamen, Handelsnamen, Warenbezeichnungen usw. in diesem Werk berechtigt auch ohne besondere Kennzeichnung nicht zu der Annahme, dass solche Namen im Sinne der Warenzeichen- und Markenschutz-Gesetzgebung als frei zu betrachten wären und daher von jedermann benutzt werden dürften.

Lektorat: Anna Pietras, Renate Schilling

Gedruckt auf säurefreiem und chlorfrei gebleichtem Papier.

Springer Gabler ist eine Marke von Springer DE. Springer DE ist Teil der Fachverlagsgruppe Springer Science+Business Media www.springer-gabler.de 


\section{Vorwort zur 2. Auflage}

Das Arbeiten mit Business Cases erweist sich - natürlich nicht unerwartet - offensichtlich als ein „Dauerbrenner-Thema“, mit einem anhaltenden Bedarf nach einer praxisorientierten, leicht verständlichen Einführung in die Welt der Wirtschaftlichkeitsrechnung von Investitionen. Diesem Bedarf soll mit der nun vorliegenden zweiten Auflage weiterhin Rechnung getragen werden.

Neben den bei jeder Neuauflage anstehenden „Renovierungsarbeiten“ wie dem Korrigieren verbliebener Irrtümer und Tippfehler, sprachlichen Umformulierungen und stellenweisen Kürzungen wurde der gesamte Text noch einmal kritisch auf logische Konsistenz und Verständlichkeit geprüft. Der inhaltliche Aufbau und die bewährte Ausrichtung des Textes als Leitfaden für die praktische Anwendung wurden nicht verändert. Inhaltliche Ergänzungen finden sich in Kapitel 10. Dort wurden einige weitere Methoden der Wirtschaftlichkeitsrechnung sowie Metriken zur Beurteilung der Vorteilhaftigkeit von Investitionen neu aufgenommen. Ebenso wurde ein Abschnitt zu Vollständigen Finanzplänen (VOFIs) ergänzt.

Die zweite Auflage möge wiederum möglichst vielen Lesern ein nützlicher Begleiter auf der Reise durch die Welt der Business Cases sein. Für Anregungen, Hinweise und Ergänzungsvorschläge bin ich natürlich nach wie vor dankbar. Sie erreichen mich unter andreas.taschner@reutlingen-university.de.

Reutlingen, im Januar 2013

Andreas Taschner 


\section{Vorwort zur 1. Auflage}

Warum lesen Sie diese Zeilen? Die Chancen sind hoch, dass Sie es tun, weil Sie demnächst selbst einen Business Case erstellen bzw. einen von anderen Personen erstellten Business Case bewerten sollen. Ein anderer Grund könnte aber auch sein, dass Sie sich schon seit längerem mit Business Cases beschäftigen und nun einfach wissen wollen, „was andere zu dem Thema so zu sagen haben".

In beiden Fällen werden Sie hoffentlich nicht enttäuscht. Das Buch versucht eine Brücke zu schlagen zwischen der vielfältigen und umfangreichen Literatur zu Investitions- und Wirtschaftlichkeitsrechnung einerseits und den in der praktischen Arbeit auftretenden Problemen andererseits. Leider überschneiden sich die auftretenden praktischen Probleme und die in der Literatur behandelten Themen und Lösungen nur zum Teil. Praktiker beklagen deshalb oft die eigenen „Wissenslücken“ bzw. umgekehrt die „Realitätsferne“ der Wissenschaft. Hier will das Buch dazu beitragen, diese Lücke zu verkleinern. Es legt den Schwerpunkt auf die in der Praxis auftretenden Probleme und versucht, dafür Lösungsverschläge aufzuzeigen - ohne aber in eine "theoriefreie Zone“ abzudriften.

Die Inhalte und Vorschläge in diesem Buch stammen zum einen aus Erkenntnissen und Erfahrungen, welche während der eigenen praktischen Beschäftigung mit dem Thema „Business Cases“ gesammelt worden sind. Sie sind deshalb natürlich teilweise subjektiv (wie jede praktische Erfahrung). Zum anderen setze ich als Lehrender an einer Hochschule aber die "akademische Brille“ (und damit den Blick auf die Theorie) nie vollständig ab. Ich hoffe, dass diese Kombination ein lesenswertes Produkt hervorgebracht hat.

Kein Werk ist so gut, dass es nicht verbessert werden könnte. Deshalb freue ich mich über jede Art des Lobes und der Kritik von Ihrer Seite. Vor allem interessiert mich natürlich der praktische Nutzen des Werkes: Finden Sie Ihre eigenen Probleme wieder? Was fehlt? Was hätten Sie zusätzlich gerne erfahren, um mit Business Cases (noch) besser umgehen zu können?

In diesem Sinne wünsche ich viel Spaß bei der Lektüre und viel Erfolg beim Erstellen der nächsten Business Cases!

Berlin, im Juli 2008

Andreas Taschner 


\section{Inhaltsverzeichnis}

Zielsetzung und Aufbau des Buches ................................................................... 11

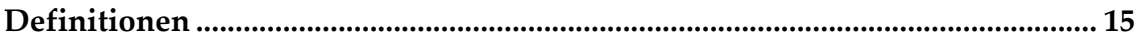

2.1 Begriffsklärung: Das Wesen eines Business Case................................................... 15

2.2 Abgrenzung von verwandten Begriffen............................................................ 17

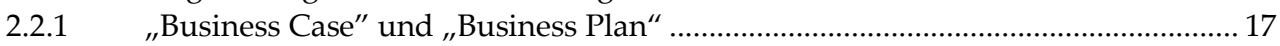

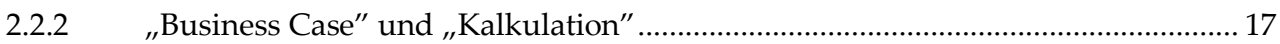

2.2.3 "Business Case" und "Kostenrechnung” .................................................................. 18

2.3 Kontrollfragen zu Kapitel 2 ........................................................................... 19

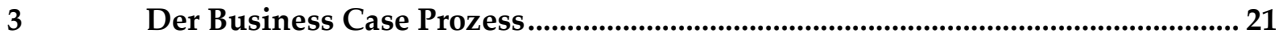

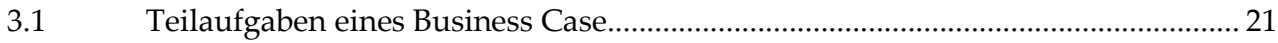

3.2 Idealtypischer Prozess einer Business Case Erstellung .......................................... 22

3.2.1 Anstoß zur Erstellung des Business Case .............................................................. 24

3.2.2 Klären der Aufgabenstellung............................................................................... 25

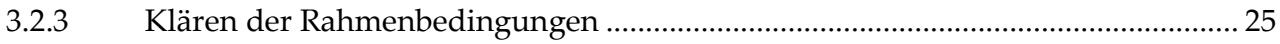

3.2.4 Bestimmung des ,richtigen“ Business Case .............................................................. 26

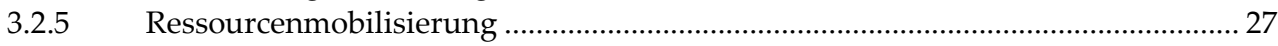

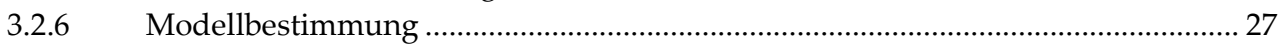

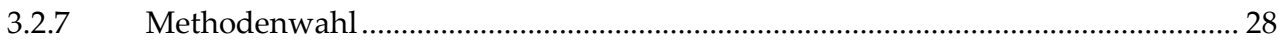

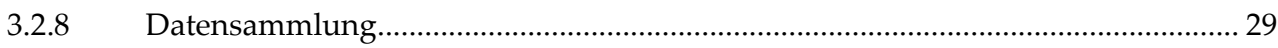

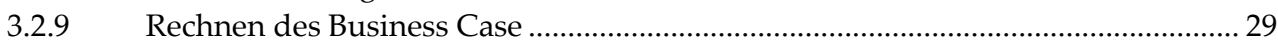

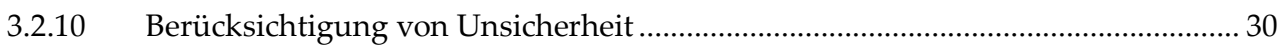

3.2.11 Analyse und Dokumentation, Präsentation .......................................................... 30

3.3 "Abkürzungen“ im Business Case Prozess ............................................................... 31

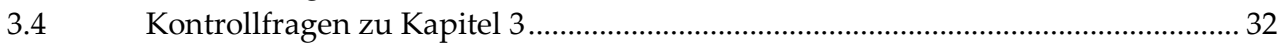

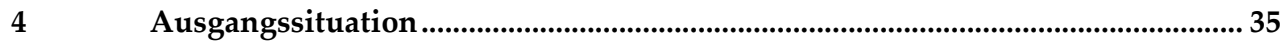

4.1 Komplexitätsdimensionen der Ausgangssituation .................................................. 35

4.2 "Business as usual“ oder „base case“ als Vergleichsbasis......................................... 38

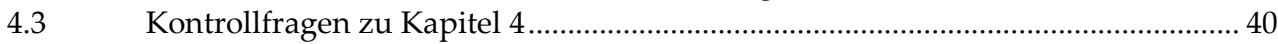

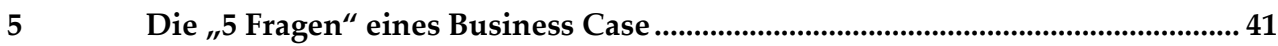

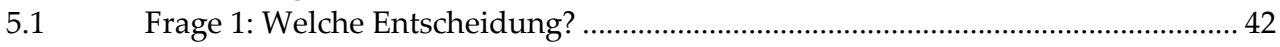

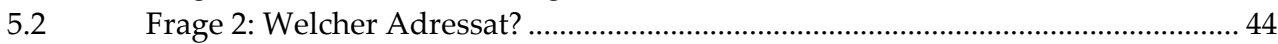

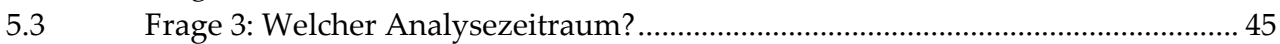

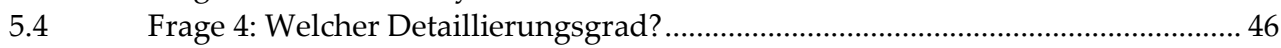

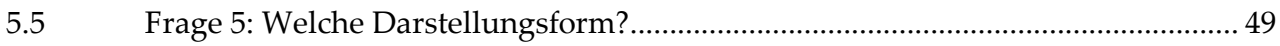

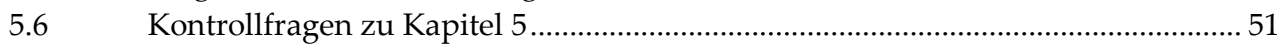




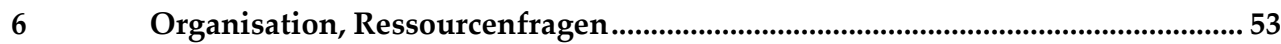

6.1 Kernkompetenzen eines Business Case Projektteams ............................................ 53

6.2 Zielkonflikte zwischen Kernkompetenzen............................................................ 55

6.3 Beschaffung der Inputdaten als organisatorische Aufgabe .................................... 57

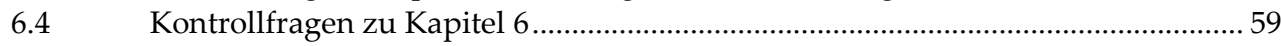

$7 \quad$ Business Case Design, Modellerstellung......................................................................61 61

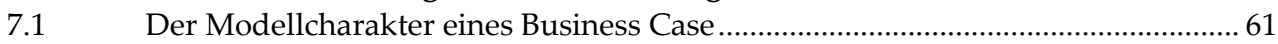

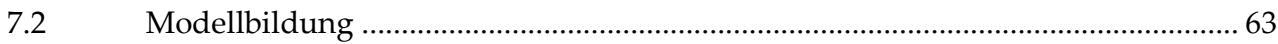

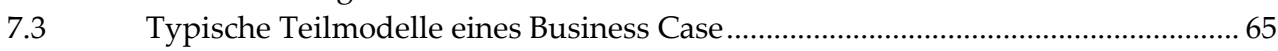

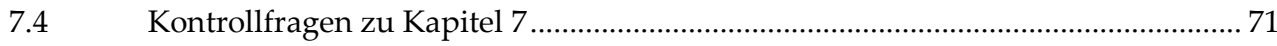

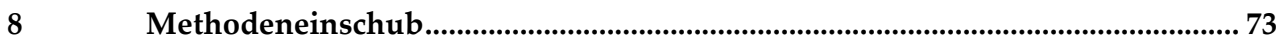

8.1 Vermögensmaximierung als Entscheidungsprämisse eines Business Case........... 73

8.2 Rechnungsgrößen eines Business Case.................................................................... 75

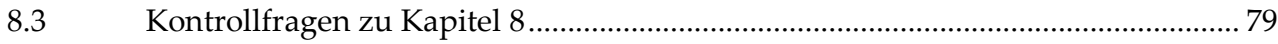

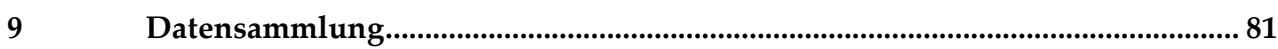

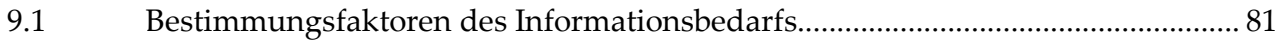

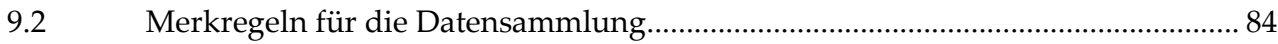

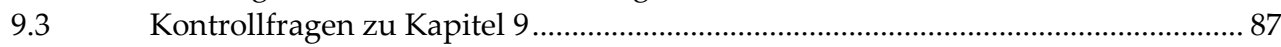

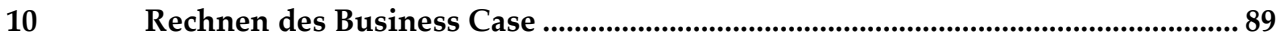

$10.1 \quad$ Zahlungsströme und ihre Vergleichbarkeit .............................................................. 89

10.2 Ein kurzer Streifzug durch die Wirtschaftlichkeitsrechnung ................................. 91

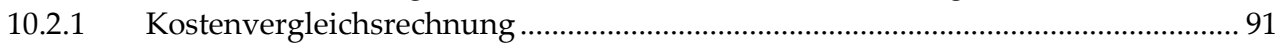

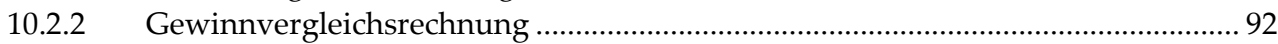

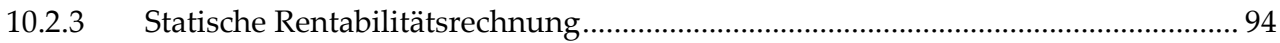

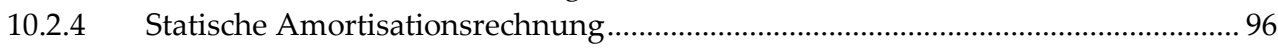

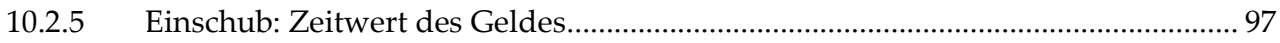

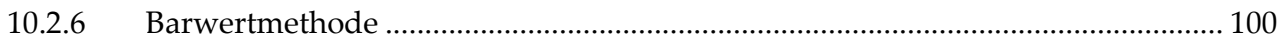

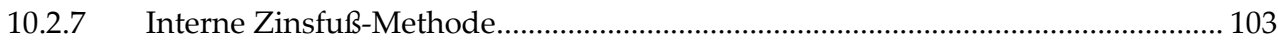

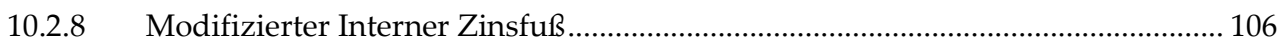

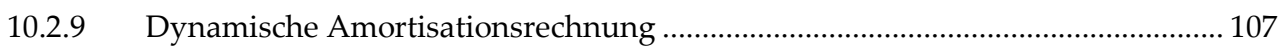

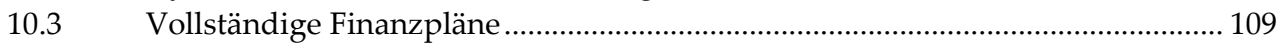

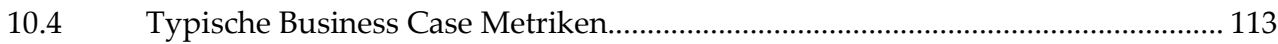

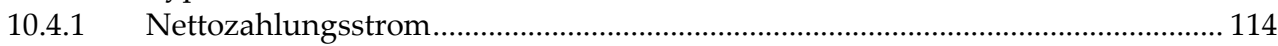

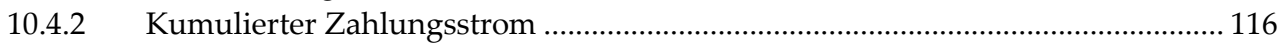

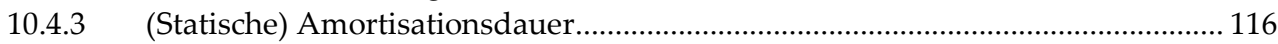

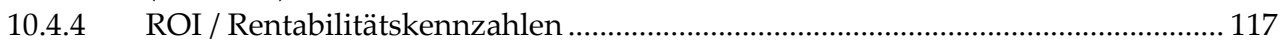

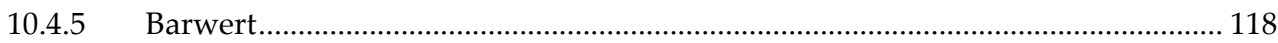

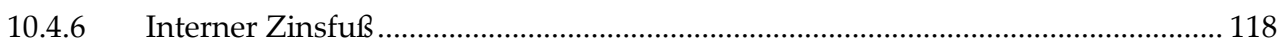

10.4.7 Benefit-to-Cost Ratio (Kosten-Nutzen-Verhältnis) ................................................ 119

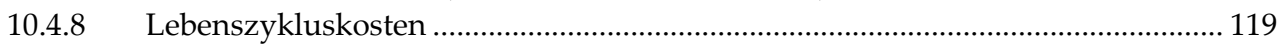




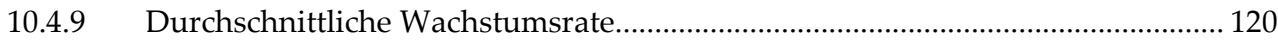

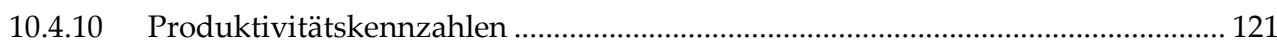

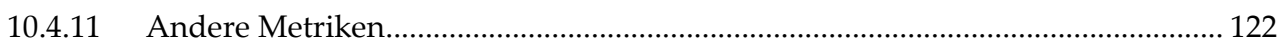

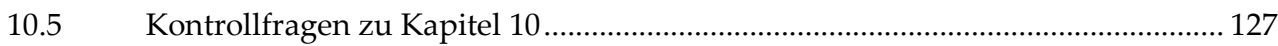

11 Berücksichtigung von Unsicherheit................................................................ 129

11.1 Alternative Verfahren zur Berücksichtigung von Unsicherheit........................... 129

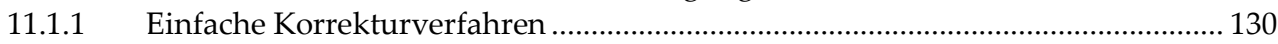

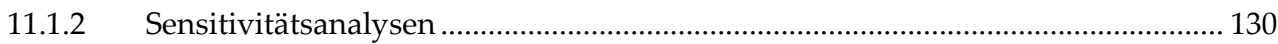

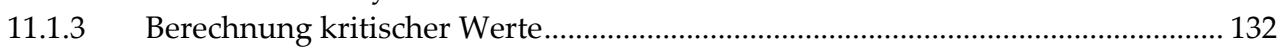

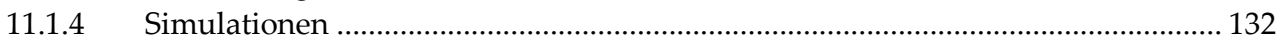

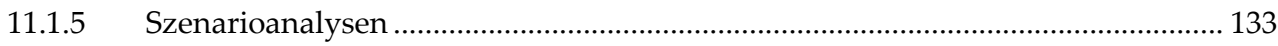

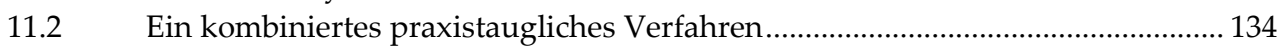

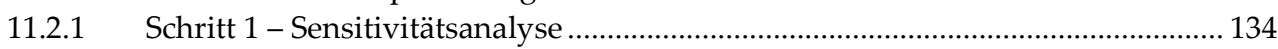

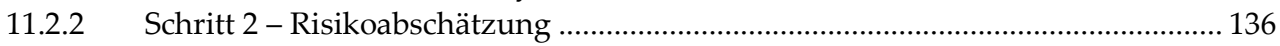

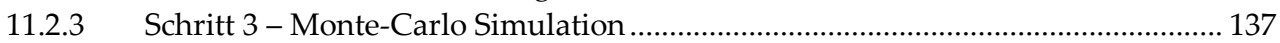

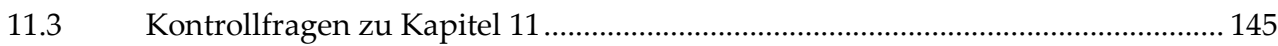

12 Weitergehende Probleme beim Rechnen eines Business Case ........................... 147

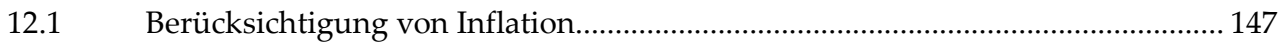

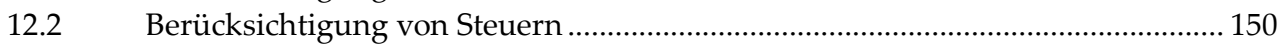

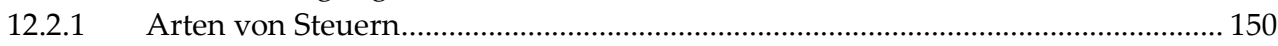

12.2.2 Grundsätzliche Berücksichtigung von Gewinnsteuern....................................... 151

12.2.3 Grenzen der Berücksichtigung von Gewinnsteuern ............................................ 152

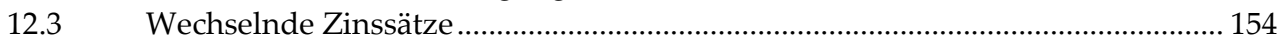

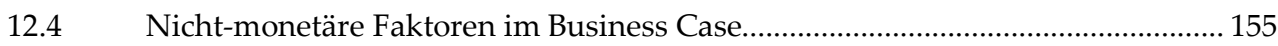

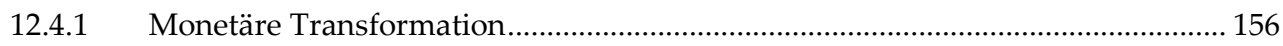

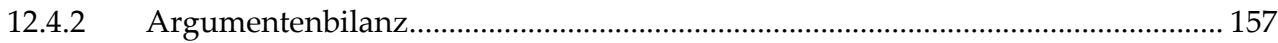

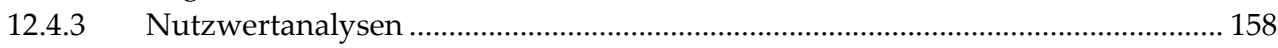

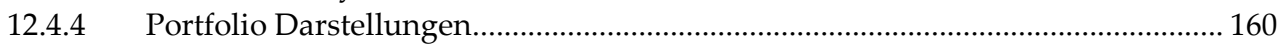

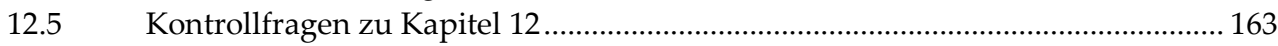

13 Darstellung und Vorstellung des Business Case ................................................... 165

13.1 Zur Notwendigkeit der Präsentation von Business Cases ..................................... 165

13.2 Tipps zur Dokumentation und Präsentation ......................................................... 169

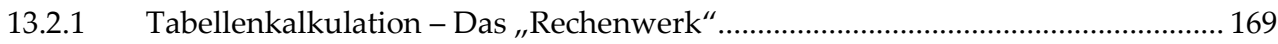

13.2.2 Bericht - Der erklärende Textteil ......................................................................... 171

13.2.3 Präsentation - Die multimediale Aufbereitung .................................................. 172

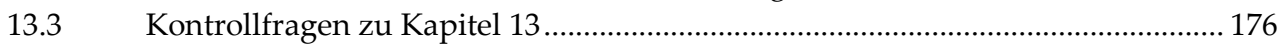

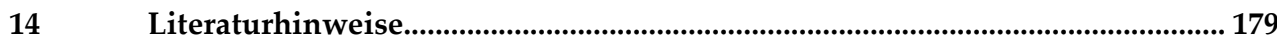




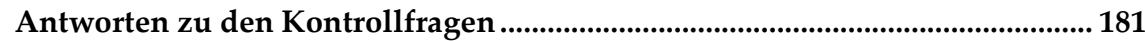

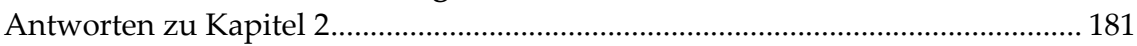

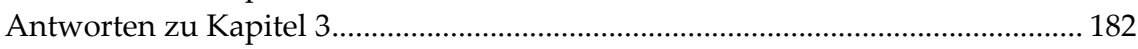

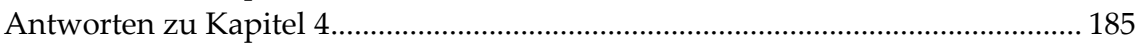

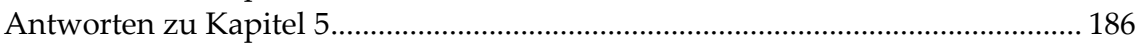

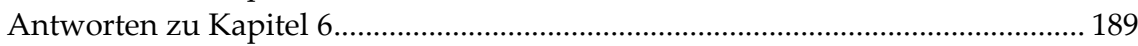

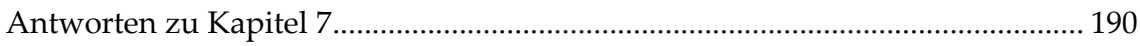

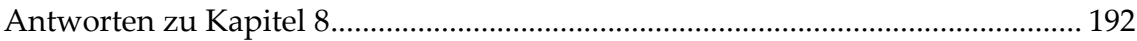

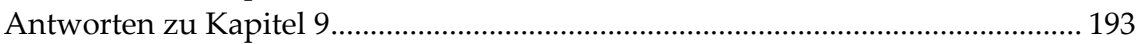

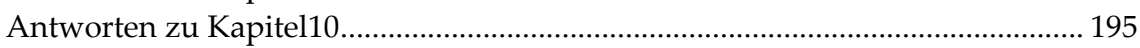

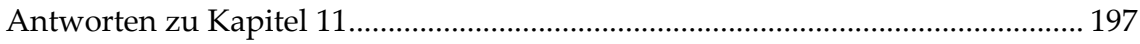

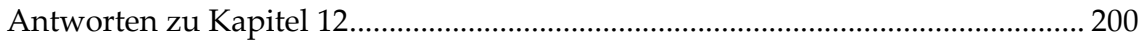

Antworten zu Kapitel 13 ................................................................................... 202 